\title{
Inhibition of connexin 43 induces hearing loss in postnatal mice
}

\author{
J. WANG and Q. SONG*
}

Department of Otolaryngology, Weifang City Hanting District People’s Hospital, Weifang 261100, Shandong, China

Received: August 6, 2020 • Accepted: December 9, 2020

Published online: March 26, 2021

(C) 2021 Akadémiai Kiadó, Budapest

\begin{abstract}
Background: Connexin $43(\mathrm{Cx} 43)$ is the most ubiquitously expressed member of the family of connexins, constituting gap junctions and mediating cell communication, still its role in hearing loss has been little studied. Methods: Immunohistochemistry was used to detect the expression pattern of Cx43. Spiral ganglia neurons (SGNs) and Corti co-culture were utilized to assay the re-innervation of hair cells by newborn SGNs. Gap19 was utilized to inhibit Cx43 hemichannels. Auditory brainstem responses (ABR) and endocochlear potential (E.P.) were measured to confirm the hearing loss. Results: The expression of Cx43 in P14 mice was higher than in P0 and P28 (adult) mice, the earlier time point coinciding with the early inner ear development. Additionally, the growth and synapse generation of fibers were inhibited after Gap 19 treatment of the co-cultures of the Corti and SGNs from newborn mice. Furthermore, the inhibition of $\mathrm{Cx} 43$ could increase the ABR threshold and decrease E.P. level in postnatal mice, whereas such an effect was not observed in adult mice. Conclusion: The function of $\mathrm{Cx} 43$ is critical during the early development of mouse cochlea but is dispensable in adult mice.
\end{abstract}

\section{KEYWORDS}

connexin 43 , hearing-loss, cochlear, spiral ganglia neurons, hair cells

\section{INTRODUCTION}

According to the World Health Organization report, the estimated incidence of disabling hearing loss is approximately $5.3 \%$ in the world's population [1]. Loss of hearing has been

\footnotetext{
*Corresponding author. Tel: +86 13406608039. E-mail: songquanfa2020@sina.com
} 
demonstrated to affect children's academic, behavioral, cognitive development, and overall quality of life $[2,3]$. Various novel methods have been attempted to treat hearing loss, including gene therapy [4]. However, the treatment is far from satisfactory. The precise biochemical, molecular, and intercellular signaling mechanisms in both normal-state hearing function and pathological processes are unknown. A total of 121 non-syndromic hearing loss genes have been identified as indicated by the Hereditary Hearing Loss Homepage. Although non-syndromic deafness is genetically heterogeneous, connexin (Cx)-26 is responsible for about $50 \%$ of nonsyndromic deafness, which indicates the importance of deciphering the relative role of the connexin family.

Twenty-one genes are known to be involved in the connexin family, which are embedded in the lipid bilayers and function as transmembrane proteins to form gap junctions between adjacent cells and mediate communication between both sides of the membrane [5]. Recent experiments indicate that connexin-associated congenital deafness can be attributed to cochlear developmental disorders rather than endocochlear potential reduction and hair cell degeneration [6]. The role of the most ubiquitously expressed component in gap junctions, Cx43 in inner ear development and function is not confirmed, even controversial. A preliminary study indicates that $\mathrm{Cx} 43$ (GJA1) mutations are initially linked to non-syndromic autosomal recessive deafness, and some research also indicates that the mutations are located in the GJA1 pseudogene rather than in GJA1 [7].

The precise auditory site of $\mathrm{Cx} 43$-related hearing loss is indicated by $\mathrm{Cx} 43$ expression analysis. The specific localization of $\mathrm{Cx} 43$ is in spiral ganglia neurons (SGNs) and nerve endings, which reach out to the inner and outer hair cells. However, on postnatal day 14 (P14), no Cx43 can be detected on the nerve endings, but the expression of Cx43 on SGNs persists until adulthood [8,9]. The mammalian inner ear, an elegant and complex sensory organ consists of the cochlea, the vestibule, and the semicircular canals. The external sound information is transmitted to SGN on the cochlea through hair cells on Corti's organ. SGNs, these bipolar neurons are the primary neurons of auditory information conduction, which can transmit information to the acoustic center in the brain [10]. Therefore, these results suggest that $\mathrm{Cx} 43$ may play an essential role in establishing standard auditory signal transmission between SGNs and Corti in the early development of the inner ear.

In this study, co-culture of SGNs and hair cells and antibody blocking are utilized to investigate the function of $\mathrm{Cx} 43$ during the early development and adult period of mice cochlea.

\section{MATERIALS AND METHODS}

\section{Ethics statement}

C57BL/6J mice were ordered from the Shanghai Laboratory Animal Center (Shanghai, China). All the procedures were conducted according to the approved protocols and animal welfare regulation of Weifang City Hanting District People's Hospital. All animal experiments complied with the ARRIVE guidelines and were carried out in accordance with the U.K. Animals (Scientific Procedures) Act, 1986 and associated guidelines, and E.U. Directive 2010/63/EU for animal experiments. 


\section{Immunohistochemical staining}

The cochleae were isolated for immunohistochemical staining at the following stages: postnatal day 0 (P0), P14, and P28 (adult) according to the previous study [8]. After deep anesthesia with pentobarbital, mice were perfused intracardially with physiological saline, followed by ice-cold 4\% paraformaldehyde (PFA). A small hole was drilled in the apex of the bony cochlea after fenestration of the round and oval windows of the cochlea. The cochleae were post-fixed in 4\% PFA (30 min, room temperature), embedded in paraffin and sectioned (5 $\mu \mathrm{m})$. Following deparaffinization and antigen retrieval, hydrogen peroxide was added to suppress endogenous peroxidase activity before the slides were blocked with 5\% goat serum. The slides were incubated with primary antibody (CX43 at 1:100, Sigma-Aldrich, St. Louis, MO, U.S.), followed by adding polymer enhancer and enzyme-labeled anti-rabbit polymers. 3,3'-Diaminobenzidine (DBA) chromogenic substrate kit (Boster Bio, China) was employed to yield insoluble brown precipitates according to the manufacture's instruction, followed by counter-staining, dehydration, and mounting. Digital images were obtained with a microscope (Nikon 80i; Tokyo, Japan).

\section{Western blotting}

Entire cochleae were collected from the mice at the following ages: P0, P14, and P28. Ice-cold immunoprecipitation buffer was utilized to homogenize the collected tissues as previously described [11]. A Bicinchoninic Acid (BCA) Protein Assay Kit (Bio-Rad Laboratories, Hercules, CA, U.S.) was used to determine protein concentration. Fifty micrograms of protein aliquots were mixed with an equal volume of sodium dodecyl sulfate loading buffer, following by denaturation at $95{ }^{\circ} \mathrm{C}$ for $10 \mathrm{~min}$, and next separated by SDS-polyacrylamide gel electrophoresis (SDS-PAGE) (12.5\% gel) and transferred to polyvinylidene fluoride (PVDF) membranes (Bio-Rad). Membranes were blocked with 5\% non-fat dried milk (1 hour, at room temperature), incubated with anti-Cx43 antibody (1:400, Invitrogen, Carlsbad, CA, U.S.) at $4{ }^{\circ} \mathrm{C}$ overnight, washed with Tris-buffered saline with $0.1 \%$ Tween-20, and then incubated with horseradish peroxidase-conjugated (HRP) IgG for 2 hours at room temperature. Signals were detected by Amersham enhanced chemiluminescence (ECL) reagents (Bio-Rad). The relative density of the protein band was normalized to glyceraldehyde-3phosphate dehydrogenase (GAPDH) with Image J software (National Institutes of Health, Bethesda, MD).

\section{Explants of the deafferented organ of Corti}

Physical ablation was performed to dissect cochlea into the inner H.C. (IHC), outer H.C. $(\mathrm{OHC})$, and surrounding supporting cells from the newborn mice, transferred to laminin (25 $\mu \mathrm{g} / \mathrm{mL})$ and poly-L-ornithine $(0.01 \%$, Sigma-Aldrich) coated cover glass and maintained in DMEM/F12 medium supplemented with $\mathrm{N} 2$ and B27 overnight at $37{ }^{\circ} \mathrm{C}$. $10 \%$ fetal bovine serum (Sigma-Aldrich), $50 \mathrm{ng} / \mathrm{mL}$ neurotrophin-3 (Chemicon, Temecula, CA, U.S.), and $10 \mathrm{ng} /$ $\mathrm{mL}$ brain-derived neurotrophic factor (Chemicon) were added during the first day of culture, and the culture medium was refreshed every 2-3 days. 


\section{Spiral ganglion neuron isolation}

$0.25 \%$ trypsin $\left(15 \mathrm{~min}, 37^{\circ} \mathrm{C}\right.$ ) was utilized to isolate spiral ganglion neurons (SGNs) from the modiolus. DMEM/F12 with 10\% FBS was added to neutralize the trypsin, and the neurons were harvested by centrifugation. The cell pellet formed was resuspended in DMEM/F12 supplemented with N2 and B27 to make a single-cell suspension. The collected neurons were cocultured with deafferented Corti. Gap19 (50 $\mu \mathrm{M}$, Tocris Bioscience, Sussex, UK) was added to the culture medium for six days to inhibit $\mathrm{Cx} 43$ hemichannels.

\section{Immunofluorescence}

Cultures were fixed with $4 \%$ PFA at room temperature for $20 \mathrm{~min}$, permeabilized with $0.1 \%$ Triton X-100, and blocked with 5\% normal goat serum for 1 hour at room temperature. The following primary antibodies: anti-Tuj1 (Sigma-Aldrich), anti-myosin VIIa (Proteus Bioscience, Ramona, CA, U.S.), anti-PSD-95 (NeuroMab, UC Davis, CA, U.S.), anti-CtBP2 (B.D. Biosciences Pharmingen, San Diego, CA, U.S.), and anti-neurofilament (N.F.) heavy chain (Chemicon) were incubated with cultures at $4{ }^{\circ} \mathrm{C}$ overnight. Cultures were further incubated with secondary antibodies - cyanine 5-conjugated goat anti-mouse IgG1 (Thermo Fisher, Rockford, IL, U.S.), biotin-conjugated Fluor goat anti-mouse IgG2a (Thermo Fisher), Alexa Fluor 568-Streptavidin (Molecular Probes, Eugene, OR, U.S.), Alexa Fluor 647 goat anti-rabbit (Molecular Probes, Eugene, OR, U.S.), or Alexa Fluor 488 goat anti-chicken (Molecular Probes, Eugene, OR, U.S.) - for 2 h at R.T. Photomicrographs were obtained using a confocal laser scanning microscope (Leica D.M. 4500B, Allendale, NJ, U.S.).

\section{Auditory brainstem response}

After postnatal day 3, mice were continuously injected with Gap19 (300 $\mu \mathrm{g} / \mathrm{kg}$ body weight, i.c.v) for ten days. The hearing ability of Gap19-treated mice was tested at the following ages: 1month, 2-month, and 3-month. To test the hearing ability of adult mice, they were injected with Gap19 using the same dose and duration as described above. An acoustically and electrically shielded booth (Industrial Acoustics Company, Bronx, NY, U.S.) was employed to perform the test according to the previous report [12]. After anesthesia, the mice were placed in a prone position, and three detecting electrodes were implanted to record the response (the active electrode, on vertex; the reference electrode, overlying left bulla; the ground electrode, overlying right bulla). ABRs were recorded to clicks presented from 0 to $80 \mathrm{~dB}$ SPL in $5 \mathrm{~dB}$ steps at $4,8,12$, 16,24 and $32 \mathrm{kHz}$, and the lowest intensity at the whole $A B R$ waveform was defined as the threshold.

\section{Endocochlear potential (E.P.) measurement}

E.P. recording was performed according to the previous study [13]. In brief, a silver-silver chloride reference electrode was inserted into the dorsum skin. At the same time, a micropipette electrode (10-40 MO) filled with $150 \mathrm{mM} \mathrm{KCl}$ was advanced to get access to the scala media of the basal turn after windowing of the bony aperture. D.C. potential signals were magnified with an amplifier (Duo 773; World Precision Instruments, Sarasota, FL, U.S.) and recorded via an A.D. converter (PowerLab 4sp, A.D. Instruments). 


\section{Statistical analysis}

The data were presented as the mean \pm standard deviation (S.D.). Student's $t$-test or one- or two-way ANOVA analysis followed with a Tukey's post hoc test was utilized. $P$ values $<0.05$ were considered statistically significant.

\section{RESULTS}

\section{Expression pattern of $\mathrm{Cx} 43$ during early development of mouse cochlea}

In the P0 mice, Cx43 staining was observed in SGN and the surrounding synapses. The SGN and the afferent terminals innervating the cochlear inner H.C.s had stronger Cx43 staining in P14 mice versus P0 mice. However, the $\mathrm{Cx} 43$ staining in SGN and inner H.C.s was significantly lower in adult mice (P28) than in younger mice (Fig. 1A). The western blotting results were consistent with the immunohistochemical staining results. The expression of $\mathrm{Cx} 43$ in P14 mice was higher than in P0 and P28 mice (Fig. 1B and C). These results indicated that Cx43 might be involved in early inner ear development.

\section{Cx43 inhibition suppresses the growth of fibers and synaptogenesis of H.C.}

To test the effects of $\mathrm{Cx} 43$ on the re-innervation of H.C.s, the innervation of Corti and the fibers' growth and H.C.s synaptogenesis were evaluated. After co-culture with Corti, the

A

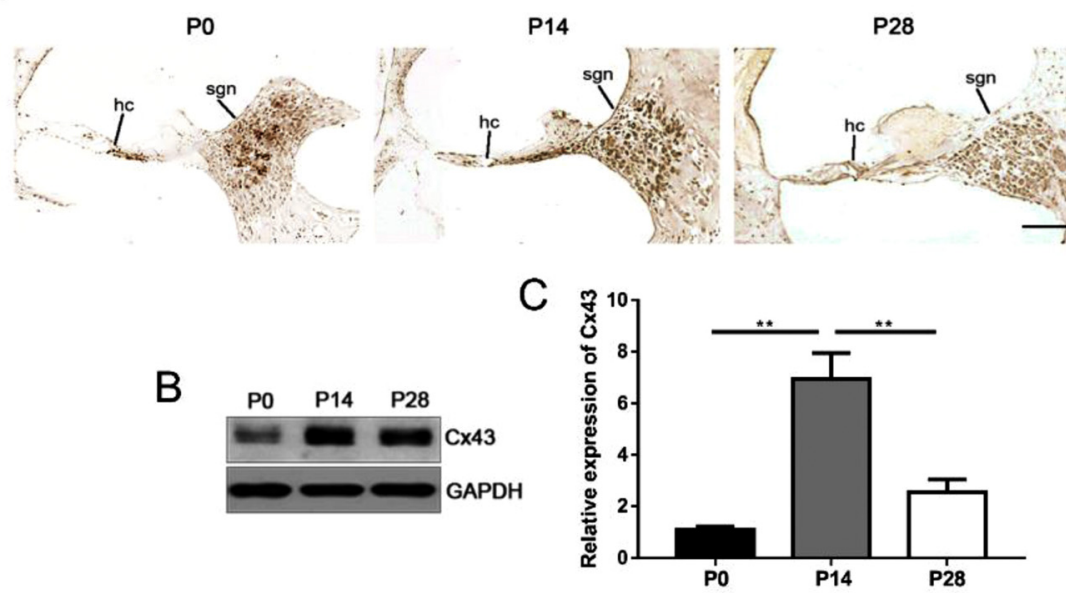

Fig. 1. Expression pattern of $\mathrm{Cx} 43$ during the early development of mouse cochlea. (A) IHC staining for Cx43 at the time points of $\mathrm{P} 0,014, \mathrm{P} 28$. (B-C) The expression of $\mathrm{Cx} 43$ was tested by western blot. Scale bars, $100 \mu \mathrm{m}$. Values were expressed as the mean \pm standard deviation. ${ }^{*} P<0.01$ with a one-way analysis of variance followed by Tukey's test. hc, hair cells. sgn, spiral ganglion neurons 
newly formed fibers derived from newborn SGNs extended and contacted the cells belonging to Corti (Fig. 2A). Cx43 blocking with Gap19 led to a decrease in the growth of fibers and a remarkable decrease in the number of inner and outer H.C.s touched by fibers from SGN (Fig. 2B). A PSD95 antibody was used to stain the postsynaptic densities, and a CtBP2 antibody was used to stain the H.C. ribbon protein. When Gsp19 was added to the co-culture system, the fiber growth was dramatically decreased (Fig. 2C and D). Meanwhile, the number of synapses per inner H.C. was significantly reduced in the Gsp19 group versus the control group (Fig. 2C and E). These results revealed that the expression of $\mathrm{Cx} 43$ mediated the growth of fibers and synaptogenesis of H.C.s.

\section{Inhibition of Cx43 elevates ABR threshold in postnatal mice}

We used ABR recording to confirm the effects of inhibition of $\mathrm{Cx} 43$ on hearing. Mean click ABR thresholds were significantly higher in the postnatal mice treated with Gap19 $(P<0.01$, Fig. 3AC). However, no significant difference was observed between the control and Gap19-treated

A

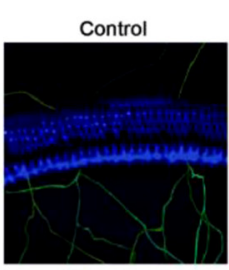

C

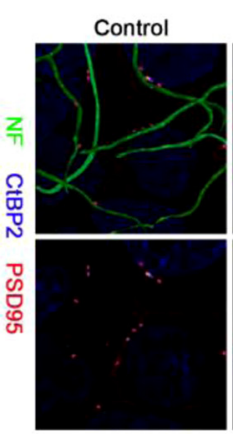

$\mathrm{B}$
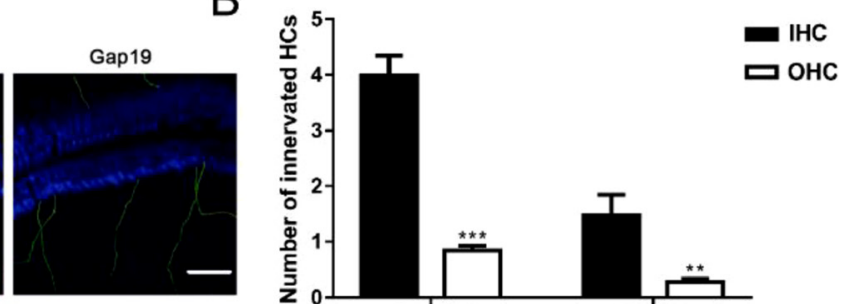

Gap19

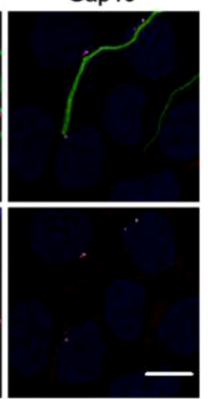

D

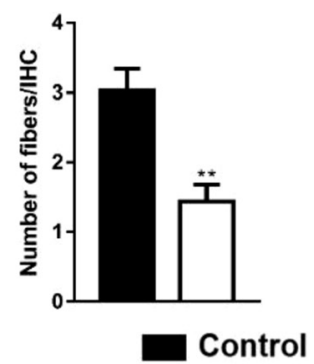

$E$

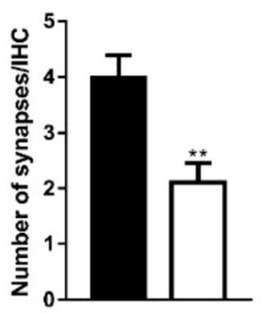

Gap19

Fig. 2. Cx43 inhibition increases fiber growth and hair cell synaptogenesis. (A) Cx43 inhibition with Gap19 treatment decreased the sprouting and growth of spiral ganglion neurons to the deafferented organ of Corti after 6 days of co-culture. Scale bars, $20 \mu \mathrm{m}$. (B) Cx43 inhibition decreased the number of inner and outer hair cells innervated by fibers from spiral ganglion neurons. (C) When Gap19 was added to the co-culture system, the fiber growth (D) and the number of new synapses generated per IHC (E) were dramatically decreased. Scale bars, $5 \mu \mathrm{m}$. Values were expressed as the mean \pm standard deviation. ${ }^{*} P<0.01$ vs control, with unpaired $t$-test 
A

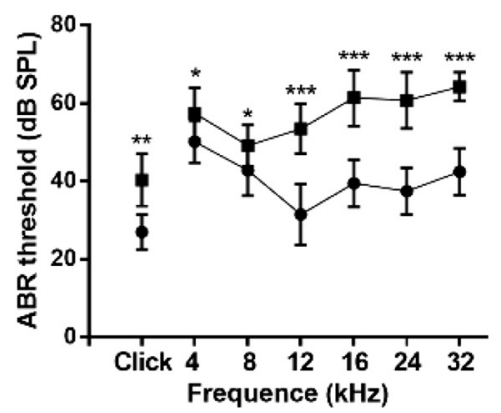

C

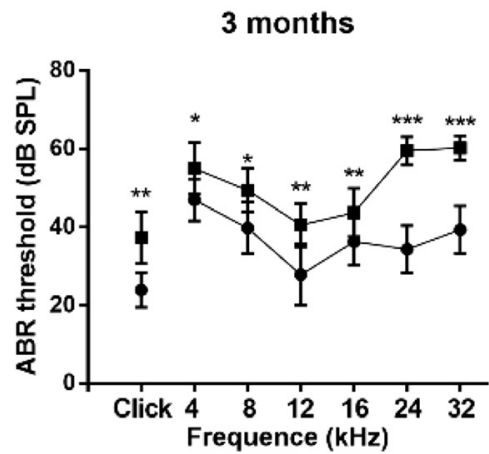

B

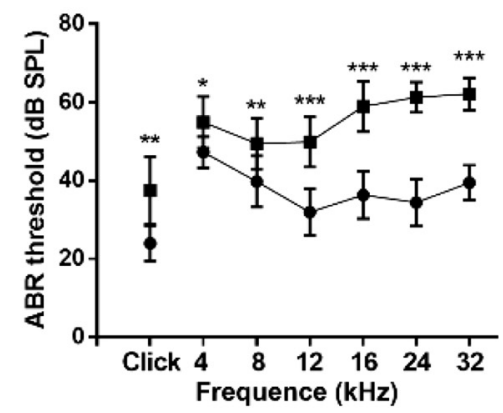

D

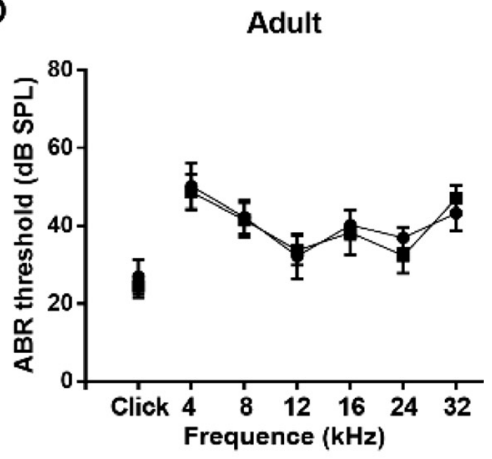

$\rightarrow$ Control

Gap19

Fig. 3. Inhibition of $\mathrm{Cx} 43$ results in hearing loss in a neonatal mouse model. (A-C) Neonatal mice (P3-P12) were injected with Gap19, and ABR analysis was performed on 1 month-, 2 month- and 3-month-old mice to assay their hearing ability. The ABR threshold of Gap19-treated mice in response to click and tone burst stimuli increased significantly compared with those in control mice. (D) Adult mice were treated with the same dose of Gap19, and ABR analysis was conducted. No difference was observed between these two groups. SPL, sound pressure level. ABR, auditory brainstem response. $n=12$. Values were expressed as the mean \pm standard deviation. ${ }^{\star} P<0.05,{ }^{* *} P<0.01,{ }^{* *} P<0.01 v s$ control with two-way ANOVA analysis and a post hoc test

adult mice (Fig. 3D). All of these results revealed that inhibition of $\mathrm{Cx} 43$ could impair hearing ability in postnatal but not adult mice.

\section{Inhibition of Cx43 suppresses E.P. level in postnatal mice}

E.P. recording in the postnatal mice was performed after Gap19 injection or without injection. After treatment with Gap19, the 1-month-old mice had significantly lower E.P. levels compared to the control (Fig. 4). The E.P. levels in the mice at 2-month and 3-month ages were significantly lower versus the control. These results indicated that the inhibition of $\mathrm{Cx} 43$ contributed to the impairment of the E.P. levels. 


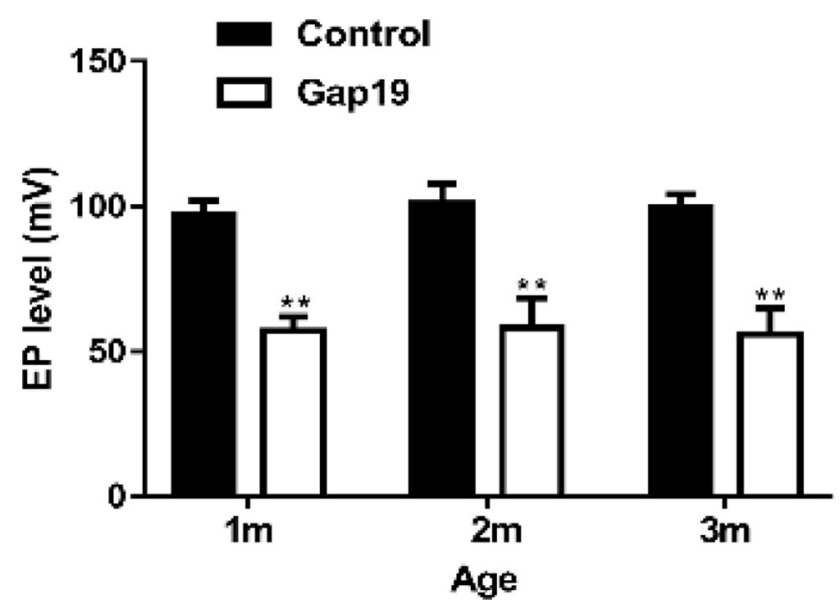

Fig. 4. E.P. levels in Gap19-treated and untreated cochleae at the age of 1 month, 2 months, and 3 months post-treatment. E.P. levels in Gap19-treated animals were significantly lower than those in controls. $n=12$. Values were expressed as the mean \pm standard deviation. ${ }^{* *} P<0.01 v s$ control with unpaired $t$-test. E.P., endocochlear potential

\section{DISCUSSION}

This investigation testifies that the function of $\mathrm{Cx} 43$ is critical during the early development of mouse cochlea. Additionally, the growth of fibers and synapse generation are inhibited after coculture of the deafferented organ of Corti and SGNs from newborn mice treated with Cx43specific inhibitor in vitro. Furthermore, the inhibition of $\mathrm{Cx} 43$ can elevate the ABR threshold and lower the E.P. level in postnatal mice, but there is no effect on adult mice.

As ubiquitous membrane proteins, connexins (Cxs) act as building blocks of the G.J.s. Thus, analysis of the cellular expression pattern and the time course of development could provide a better understanding of the molecular basis of hereditary deafness [14]. Cx43 expression is found in the developing cochleae of mice as early as at embryonic stages [15]. The bony layer of the otic capsule starts to have $\mathrm{Cx} 43$ expression from P8, and the expression increases with maturation [16]. In the present study, we find that $\mathrm{Cx} 43$ expression changes remarkably during the postnatal development period from P0 to P14, and decreases later, at the adult stage (P28), which is consistent with cDNA microarray findings showing that the Cx43 RNA transcription level peaks at P10 and then falls dramatically starting from P12 [16]. All of these data indicate that the 10-day postnatal period is critical for cochlear maturation; therefore, the expression pattern of $\mathrm{Cx} 43$ may be pivotal for cochlear growth during cochlear development.

To evaluate the effects of Cx43 expression on hearing development, Gap19, a Cx43 hemichannel blocker was used in the in vitro SGN and H.C. co-culture system as mentioned before. In the current study, we find that IHC, OHC, fibers per IHC, and synapses per IHC in the Gap19-treated cultures are fewer versus the control groups. These findings may indicate that the inhibition of $\mathrm{Cx} 43$ can inhibit the growth of fibers and the synaptogenesis of H.C.s; thus, the function of $\mathrm{Cx} 43$ may be associated with the spiral ganglion neurons' proliferation and neuroprotection, which is consistent with previous reports [17]. Although the neuroprotective role 
of $\mathrm{Cx} 43$ has been demonstrated in numerous in vitro studies $[18,19]$, the neuroprotection resulting from the expression of $\mathrm{Cx} 43$ is not observed in heterozygote $\mathrm{Cx} 43$ null mice [19]. Our in vitro investigation indicates that $\mathrm{Cx} 43$ may play a vital role in establishing standard auditory signal transmission between SGN and H.C.s during early inner ear development and maturation.

To confirm the influence of $\mathrm{Cx} 43$ on hearing ability in vivo, the ABR threshold and E.P. level were measured. In the ABR threshold evaluation, we also find that the ABR threshold increases in the mice treated with Gap19 in the neonatal mouse model, indicating that Cx43 blocking may result in hearing loss, and Cx43 may play a key role during hearing development. In contrast, no such function was observed in adult mice. Moreover, the expression of Cx43 dramatically decreased in adult mice compared to the P14 mice. Therefore, the expression of Cx43 can mediate hearing ability. In the adult mice, there is little Cx43 expression; thus, the inhibition of $\mathrm{Cx} 43$ has few effects on hearing ability. However, in the postnatal mice, the expression of Cx43 increased with increasing age. After inhibiting $\mathrm{Cx} 43$ expression, hearing loss was observed in the postnatal mice; thus, the expression of $\mathrm{Cx} 43$ plays a pivotal role during the early cochlear development but not in the mature cochlea.

In the in vivo E.P. level evaluation, we find that inhibition of $\mathrm{Cx} 43$ expression can result in significantly lower E.P. levels. Additionally, we also find that the expression of Cx43 in both SGN and inner H.C. increases with increasing postnatal days. After treatment with Gap19, the expression of $\mathrm{Gx} 43$ is inhibited. An alternation of $\mathrm{Cx} 43$ might result in changes in potassium buffer capacity, which can lower sensory thresholds and membrane physiological potential changes [20]. Furthermore, the potassium influx from the endolymph can excite H.C.s, and the E.P. enhances the sensitivity of H.C.s [21-23]. The perilymph and endolymph are the two types of extracellular fluid that fill the cochlea of the inner ear. The apical and basolateral surfaces of H.C.s are exposed to endolymph and perilymph, respectively [24]. Therefore, the potassium concentration of the endolymph can determine the maturation of the E.P. levels during postnatal cochlea development, and suppressing the E.P. can result in hearing loss [17]. Cx43 has been demonstrated to participate in potassium recycling to the cochlear endolymph [25]. The present findings demonstrate that the expression of $\mathrm{Cx} 43$ is responsible for the regulation of the E.P. level, presumably by mediating the potassium influx from the endolymph.

\section{CONCLUSION}

The present results demonstrate that $\mathrm{Cx} 43$ expression does affect inner ear development, it plays a critical function in developing and maintaining auditory function in postnatal mice, but there is no impact on adult mice.

Conflicts of interest: The authors declare that they have no conflict of interest.

Funding: None.

\section{ACKNOWLEDGMENTS}

None. 


\section{REFERENCES}

1. Crowson MG, Hertzano R, Tucci DL. Emerging therapies for sensorineural hearing loss. Otol Neurotol 2017; 38(6): 792-803.

2. Wake M, Hughes EK, Poulakis Z, Collins C, Rickards FW. Outcomes of children with mild-profound congenital hearing loss at 7 to 8 years: a population study. Ear and Hearing 2004; 25(1): 1-8.

3. Nasralla HR, Gomez MVSG, Magalhaes AT, Bento RF. Important factors in the cognitive development of children with hearing impairment: case studies of candidates for cochlear implants. Int Arch Otorhinolaryngol 2014; 18(4): 357-61.

4. Wei C, He Z, Kong W. Application of gene therapy in auditory system diseases. STEMedicine 2020; 1(1).

5. Pohl U. Connexins: key players in the control of vascular plasticity and function. Physiol Rev 2020; 100(2): 525-72.

6. Wingard JC, Zhao H-B. Cellular and deafness mechanisms underlying connexin mutation-induced hearing loss - a common hereditary deafness. Front Cell Neurosci 2015; 9: 202-202.

7. Paznekas WA, Boyadjiev SA, Shapiro RE, Daniels O, Wollnik B, Keegan CE, et al. Connexin 43 (GJA1) mutations cause the pleiotropic phenotype of oculodentodigital dysplasia. Am J Hum Genet 2003; 72(2): 408-18.

8. Liu W, Yang J. Preferentially regulated expression of connexin 43 in the developing spiral ganglion neurons and afferent terminals in post-natal rat cochlea. Eur J Histochem EJH 2015; 59(1).

9. Kim AH, Nahm E, Sollas A, Mattiace L, Rozental R. Connexin 43 and hearing: possible implications for retrocochlear auditory processing. The Laryngoscope 2013; 123(12): 3185-93.

10. Jovanovic S, Milenkovic I. Purinergic modulation of activity in the developing auditory pathway. Neurosci Bull 2020; 36: 1285-98.

11. Shen X, Dong Y, Xu Z, Wang H, Miao C, Soriano SG, et al. Selective anesthesia-induced neuroinflammation in developing mouse brain and cognitive impairment. Anesthesiol: J Am Soc Anesthesiologists 2013; 118(3): 502-515.

12. Akil O, Oursler AE, Fan K, Lustig LR. Mouse auditory brainstem response testing. Bio Protoc 2016; 6(6): e1768.

13. Kada S, Hamaguchi K, Ito J, Omori K, Nakagawa T. Bone marrow stromal cells accelerate hearing recovery via regeneration or maintenance of cochlear fibrocytes in mouse spiral ligaments. The Anatomical Rec 2020; 303(3): 478-86.

14. Cai WJ, Wang ZL, Wei CF, Wu M, Zheng WP, Zhang HM, et al. Prognostic evaluation of NANOG and OCT4 expression for posttransplantation hepatocellular carcinoma recurrence. J Cell Biochem 2019; 120(5): 8419-29.

15. Cohen-Salmon M, Maxeiner S, Krüger O, Theis M, Willecke K, Petit C. Expression of the connexin43-and connexin45-encoding genes in the developing and mature mouse inner ear. Cell Tissue Res 2004; 316(1): 15-22.

16. Ahmad S, Chen S, Sun J, Lin X. Connexins 26 and 30 are co-assembled to form gap junctions in the cochlea of mice. Biochem Biophysical Res Commun 2003; 307(2): 362-8.

17. Walters BJ, Zuo J. Postnatal development, maturation and aging in the mouse cochlea and their effects on hair cell regeneration. Hear Res 2013; 297: 68-83.

18. Lin JH-C, Lou N, Kang N, Takano T, Hu F, Han X, et al. A central role of connexin 43 in hypoxic preconditioning. J Neurosci 2008; 28(3): 681-95. 
19. Nakase T, Söhl G, Theis M, Willecke K, Naus CC. Increased apoptosis and inflammation after focal brain ischemia in mice lacking connexin43 in astrocytes. The Am J Pathol 2004; 164(6): 2067-75.

20. Cherkas PS, Huang T-Y, Pannicke T, Tal M, Reichenbach A, Hanani M. The effects of axotomy on neurons and satellite glial cells in mouse trigeminal ganglion. Pain 2004; 110(1-2): 290-8.

21. Hudspeth AJ. How the ear's works work. Nature 1989; 341(6241): 397-404.

22. Hibino H, Kurachi Y. Molecular and physiological bases of the $\mathrm{K}+$ circulation in the mammalian inner ear. Physiology 2006; 21(5): 336-45.

23. Kusakari J, Ise I, Comegys T, Thalmann I, Thalmann R. Effect of ethacrynic acid, furosemide, and ouabain upon the endolymphatic potential and upon high energy phosphates of the stria vascularis. The Laryngoscope 1978; 88(1): 12-37.

24. Guo W, Lei C, Dengke L, Wei S, Shiming Y. Endocochlear potential and potassium concentration recording in minipig cochlea. J Otology 2012; 7(2): 103-5.

25. Liu XZ, Xia XJ, Adams J, Chen ZY, Welch KO, Tekin M, et al. Mutations in GJA1 (connexin 43) are associated with non-syndromic autosomal recessive deafness. Hum Mol Genet 2001; 10(25): 2945-51. 